CLINICAL STUDY

\title{
Follicular thyroid neoplasms can be classified as low- and high-risk according to HBME-1 and Galectin-3 expression on liquid-based fine-needle cytology
}

\author{
Guido Fadda ${ }^{1}$, Esther Diana Rossi ${ }^{1}$, Marco Raffaelli ${ }^{3}$, Alfredo Pontecorvi ${ }^{2}$, Stefano Sioletic ${ }^{1}$, Francesca Morassi ${ }^{1}$, \\ Celestino Pio Lombardi ${ }^{3}$, Gian Franco Zannoni ${ }^{1}$ and Guido Rindi ${ }^{1}$ \\ Divisions of ${ }^{1}$ Anatomic Pathology and Histology, ${ }^{2}$ Endocrinology and ${ }^{3}$ Endocrine Surgery, Agostino Gemelli' School of Medicine, Catholic University of \\ Sacred Heart, Largo Francesco Vito, 1 - 00168 Rome, Italy
}

(Correspondence should be addressed to G Fadda; Email: guidofadda@rm.unicatt.it)

\begin{abstract}
Design: Fine-needle aspiration biopsy (FNAB) is the most reliable diagnostic tool in the diagnosis of thyroid nodules. A cytologic diagnosis of follicular neoplasm with atypical cells of undetermined significance (FN/AUS) implies that the selection of patients between surgery and follow-up is difficult. In this setting immunocytochemical stainings might be helpful. The efficacy of a panel made up of HBME-1 and Galectin-3 antibodies is evaluated in cases processed by liquid-based cytology (LBC). Methods: Out of 7091 thyroid FNAB processed by LBC method, 120 cases undergoing surgery successively were selected. These cases were classified as benign lesion (BL, eight cases), FN, including the ACUS category of the Bethesda classification (FN/AUS, 50 cases), suspicious for malignancy (SM, 59 cases), and malignant neoplasm (MN, three cases). Immunostains for HBME-1 and Galectin-3 were carried out on the LBC slides.

Results: All MN and BL were histologically confirmed. FN/AUS and SM showed a malignancy risk of 24 and $72.9 \%$ respectively. The complete immunocytochemical panel was positive in $83.3 \%$ of the cases resulting in malignancy and negative in $87.5 \%$ of cases resulting in benign histology. Among the FN/AUS, the complete positive immunocytochemical panel was detected in $76.9 \%$ of cases resulting as malignant and the complete negative immunocytochemical panel was observed in $96.8 \%$ of cases resulting as benign at histology.

Conclusions: The expression of HBME-1 and Galectin-3 in cases classified as FN/AUS on LBC-processed FNABs can effectively distinguish lesions, which need immediate surgery (high risk or FNH or Thy $3 \mathrm{~h}$ ) from those which can be followed-up (low risk or FNL or Thy 3l).
\end{abstract}

European Journal of Endocrinology $165447-453$

\section{Introduction}

Fine-needle aspiration biopsy (FNAB) represents a valid diagnostic choice combined with clinical and ultrasound evaluation, in the characterization of thyroid nodules. It may achieve a precise interpretation of the nature of thyroid lesions in more than $70 \%$ of cases and it leads to a correct clinical strategy (mostly related to the surgical indication) in more than $90 \%$ of cases $(1,2)$. It is simple, safe and extremely cost-effective especially when performed under sonographic guidance $(3,4)$. Some problems may arise in the cytologic diagnosis of lesions exhibiting a predominant microfollicular pattern. The real nature of these lesions can be assessed only by the detection of capsular and vascular invasion whereas the needle biopsy may sample only the center of the nodule. Several studies (5-9) have investigated different morphologic features (especially the nuclear 'atypia' of the follicular cells) that may enable a distinction between the most important neoplasms, which are follicular adenoma (FA), follicular carcinoma (FC), and the follicular variant of papillary carcinoma (FVPC). However, this possibility is still speculative and the majority of authors feel that the diagnosis of indeterminate cytology is still unavoidable.

In thyroid pathology, immunohistochemistry has been used for the differential diagnosis of follicular and $\mathrm{C}$ cell-derived neoplasms. Recently, the expression of markers of malignancy has also been used as an additional tool for diagnosing malignant tumors, regardless of the presence of capsular or vascular invasion (10).

Among these markers of malignancy HBME-1 and Galectin-3 have reached high values of specificity and sensitivity in discriminating benign lesions (BL) from malignancies.

Galectin-3 is a component of the $\beta$-galactosidebinding lectins whose function is still unclear. It appears 
to be involved in the cell-cell and cell-matrix modulation and, according to some authors (11), in cell growth and differentiation. Therefore, this antibody could play a role in the malignant transformation of thyroid cells and it is expressed in a high proportion of carcinomas, especially of the papillary histotype (11-13).

HBME-1 is an MAB directed against a still unknown membrane antigen of the microvillar surface of the mesothelioma cells. It yields positive in neoplastic follicular cells, mostly from papillary carcinoma, and negative in $\mathrm{BL}(14,15)$.

However, none of them has shown a diagnostic accuracy sufficient for using as single antibody in the diagnosis of malignant thyroid neoplasms $(10,16)$.

Immunocytochemistry may find new application fields in fine-needle aspiration cytology, especially when a diagnosis of follicular neoplasm (FN/atypical cells of undetermined significance (AUS) - Thy 3) is made $(4,5)$. The latter category accounts for $10-25 \%$ of all cases and represents a therapeutic problem because of the low, though not irrelevant, risk of malignancy. Therefore, the cases diagnosed as such are often addressed to surgery that results in a large amount of inappropriate thyroidectomies. In fact, about $80 \%$ of all thyroid operations for FN/AUS yield a histologic diagnosis of benign nodule (5-8).

Immunocytochemical techniques can be applied on liquid-based cytology (LBC) (17-19). This technique has been introduced to obtain thin-layer smears in cervical cytology. The excellent results in this field prompted a wider application to virtually all cytological branches, including thyroid FNAB (20-27). The current investigation has been carried out to evaluate the effectiveness of a panel made up by HBME-1 and Galectin-3 on LBC in identifying two subsets of FN/AUS with low and high malignant risk that might be managed differently to achieve a reduction of inappropriate surgery.

\section{Materials and methods}

During the period August 1, 2006-December 31, 2008, 7091 thyroid FNAB were carried out in the Divisions of Endocrine Surgery, Endocrinology and Anatomic Pathology and Histology of the Catholic University, 'Agostino Gemelli' School of Medicine and Hospital of Rome. The diagnoses of FN/AUS were $953(13.4 \%)$ and those of suspicious for malignancy (SM) 227 (3.2\%). The series under investigation included 120 cases, 50 diagnosed as FN (FN/AUS), corresponding to Thy 3 of the BTA classification (28), which also includes oxyphilic neoplasms of the NCI Bethesda classification (9) and 59 lesions classified as SM (Thy 4 of BTA). The remaining 11 cases included eight cases diagnosed as non-neoplastic lesion (Thy 2 of the BTA classification) and three papillary carcinomas, which were used as controls. The following inclusion criteria were applied: i) all cytologic diagnoses reviewed by an experienced pathologist (G F); ii) presence of abundant material so that immunocytochemical stainings could be carried out; and iii) availability of the definitive histology specimen. The morphologic criteria for the inclusion of each case processed by LBC in the corresponding cytologic category were presented in previous papers of our group $(26,27)$. The series included 35 male and 85 female patients with a median age of 32.5 years (range 13-78 years). All aspirations were performed with 25-27G needles under sonographic guidance with at least two until four consecutive passes for each lesion (4).

After the aspiration, the needle was rinsed in Cytolit solution and then the sample was processed by the ThinPrep 2000 method (Hologic Cytyc Co., Marlborough, MA, USA), fixed with 95\% ethyl alcohol and stained with Papanicolaou.

Those cases showing a difficult cytologic picture were discussed by three cytopathologists (E D R, G F, and G F Z) until a final agreement was achieved.

Immunocytochemical staining was carried out with the avidin-biotin-peroxidase complex on LBC slides using the following antibodies: HBME-1 (Dako, Glostrup, Denmark, 1:100 dilution) and Galectin-3 (Ventana, Tucson, AZ, USA, 1:100 dilution). The slides were washed three times in PBS and then preincubated in normal serum with PBS (1:50) for 20 min before incubating at $4{ }^{\circ} \mathrm{C}$ with the primary antibody. Then the slides were washed three times with PBS and incubated with the biotinylated secondary antibody conjugated with the avidin-biotin-peroxidase complex. The reaction was developed using 3,3'-diamino-benzidine. All slides were counterstained with hematoxylin for $5 \mathrm{~s}$, rinsed in water three times then mounted for the microscopic examination. The positivity was assessed, for each cytological case, when at least $50 \%$ of follicular cells showed a convincing cytoplasmic positivity. The cytodiagnostic groups of SM (Thy 4) and malignant neoplasm (MN) (Thy 5) were referred to surgery according with the BTA and ATA guidelines (29) whereas the BL (Thy 2) cases were operated on when esthetic or functional problems occurred.

For the FN (Thy 3) category, which shows a relevant risk of carcinomatous occurrence, the same guidelines

Table 1 Cytohistological comparison (120 cases).

\begin{tabular}{llllc}
\hline & \multicolumn{3}{c}{ Histological diagnosis } \\
\cline { 2 - 5 } $\begin{array}{l}\text { Cytological } \\
\text { diagnosis }\end{array}$ & Benign & FA & Malignant & Overall \\
\hline BL: Thy 2 & $8(100 \%)$ & 0 & 0 & 8 \\
FN/AUS: Thy 3 & $20(40 \%)$ & $19(38 \%)$ & $11(22 \%)$ & 50 \\
SM: Thy 4 & $6(10.2 \%)$ & $10(16.9 \%)$ & $43(72.9 \%)$ & 59 \\
MN: Thy 5 & 0 & 0 & $3(100 \%)$ & 3 \\
\hline
\end{tabular}

BL, benign lesion; FA, follicular adenomas; FN, follicular neoplasm; SM, suspicious of malignancy; MN, malignant neoplasms. 
Table 2 Cytohistologic comparison for the immunocytochemical results (all 120 cases).

\begin{tabular}{llll}
\hline & \multicolumn{3}{c}{ Histological diagnosis } \\
\cline { 2 - 4 } Immunocytochemistry & Benign $^{\mathrm{a}}$ & Malignant & Overall \\
\hline Concordant negative & $42(87.5 \%)$ & $6(12.5 \%)$ & 48 \\
HBME-1+, Galectin-3- & $10(45.4 \%)$ & $12(54.6 \%)$ & 22 \\
HBME-1-, Galectin-3+ & $2(100 \%)$ & 0 & 2 \\
Concordant positive & $8(16.7 \%)$ & $40(83.3 \%)$ & 48 \\
\hline
\end{tabular}

ancluding follicular adenomas; $P<0.001$.

suggest that surgery is routinely advised after a multidisciplinary discussion.

The surgical specimens were fixed in 10\% buffered formaldehyde, embedded in paraffin and the $5 \mu \mathrm{m}$ thick sections were stained with hematoxylin-eosin.

Immunostains for the above-mentioned antibodies, particularly in those cases that did not show a complete positivity of the panel in the preoperative cytology, were carried out also on the histological sections. It was performed by the $\mathrm{ABC}$ method with the same antibodies used for the immunostains on LBC slides.

\section{Statistical analysis}

Sensitivity, specificity, and overall accuracy of FNAB were calculated as follows: the BL category (Thy 2) were defined as benign, the categories FN/AUS and SM (Thy 3 and 4), were included in the malignant lesions. Inadequate specimens and microscopic carcinomas incidentally found in the final pathologic examination were excluded and the statistical analysis was performed considering only the histological diagnosis of the sampled lesion.

Benign and malignant lesions were compared for cytological and immunocytochemical pattern. Statistical analysis was performed by a commercially available statistic software package (SPSS 10.0 for Windows SPSS, Inc., Chicago, IL, USA). The $\chi^{2}$ test was used for categorical variables up to 6 degrees of freedom. A $P$ value $<0.05$ was considered as significant.

All patients had been appropriately informed regarding the use of LBC method for processing their aspiration samples and a written informed consent was signed by each of them.

\section{Results}

The 120 cytological cases were diagnosed as follows: 8 BL (Thy 2), 50 FN/AUS, which did not include any oxyphilic neoplasm (Thy 3); 59 SM (Thy 4), 3 papillary carcinomas (PC) (Thy 5). All cases underwent surgery and the histologic examination confirmed all three PC (one showing an insular pattern) and all BL (three revealed FAs at histology). Of the $50 \mathrm{FN} / \mathrm{AUS}, 11$ (22\%) resulted as carcinoma at histology (ten papillary carcinomas and one FC, Table 1). Forty-three out of 59 cases of SM $(72.9 \%)$ were malignant; all but one (which was an insular carcinoma arising from a papillary carcinoma) was diagnosed as PC.

The application of the immunocytochemical stainings to the ThinPrep 2000 processed material showed that 96 cases $(80 \%)$ exhibited a concordant panel (Table 2). In the group of $\mathrm{PC}$, the complete panel was positive in all cases and, accordingly, within the benign group all cases were negative for HBME-1 and Galectin3. In the FN/AUS category (Thy 3, Table 3) the concordant panel resulted positive in 13 cases (resulting three benign and ten, $76.9 \%, \mathrm{MN}$ ) and negative in 31 cases (30 resulted benign neoplasms, including 14 adenomas, and one, $3.2 \%$, was a PC). The results of IHC for the subgroup of SM showed a concordant positivity for HBME-1 and Galectin-3 in 32 cases (yielding five BL and $27,84.4 \%$, malignancies) and a concordant negativity in ten (five benign and five malignant, Table 4 and Fig. 1). In the latter category, the low efficacy of the immunocytochemistry compared with morphology explains the relatively low values of specificity and sensitivity for the complete series (Table 5).

Regarding the cases with discordant panel, the figures in Table 2 show that the best correlation between cytoplasmic positivity and occurrence of malignancy can be observed with HBME-1 instead of Galectin-3. In fact, out of 22 cases of HBME-1 positivity and Galectin-3 negativity 13 resulted in malignancy (59.1\%) whereas in the opposite instance both cases were benign.

The correlation of the presence of the complete panel with the histological results for the category of FN/AUS (Table 3) shows that the simultaneous negativity of both antibodies is almost always associated with a benign diagnosis whereas complete positivity is associated with an $\mathrm{MN}$ in 10 out of 13 cases $(76.9 \%)$.

The descriptive statistical analysis, depicted in Table 5, shows the differences in sensitivity, specificity, diagnostic accuracy, positive, and negative predictive values of the cytologic diagnosis alone, of the immunocytochemistry, of the category of FN/AUS (Thy 3 according to BTA guidelines) and of HBME-1 and Galectin-3 alone.

In total, 19 cases (15.8\%) were selected for the repetition of the immunostains for HBME-1 and

Table 3 Cytohistological comparison for immunocytochemical results (only FN: Thy $3 ; 50$ cases).

\begin{tabular}{lll}
\hline & \multicolumn{2}{c}{ Histological diagnosis } \\
\cline { 2 - 3 } Immunocytochemistry & Benign $^{\mathrm{a}}$ & Malignant \\
\hline HBME-1+, Galectin-3+ & $3(23.1 \%)$ & $10(76.9 \%)$ \\
HBME-1+, Galectin-3- & $5(100 \%)$ & 0 \\
HBME-1-, Galectin-3+ & $1(100 \%)$ & 0 \\
HBME-1-, Galectin-3- & $30(96.8 \%)$ & $1(3.2 \%)$ \\
Overall & $39(78 \%)$ & $11(22 \%)$ \\
\hline
\end{tabular}

ancluding follicular adenomas; $P<0.001$. 
Table 4 Cytohistological comparison for immunocytochemical results (only suspicious of malignancy diagnoses, Thy 4, 59 cases).

\begin{tabular}{lll}
\hline & \multicolumn{2}{c}{ Histological diagnosis } \\
\cline { 2 - 3 } Immunocytochemistry & Benign $^{\mathrm{a}}$ & Malignant \\
\hline HBME-1+, Galectin-3+ & $5(15.6 \%)$ & $27(84.4 \%)$ \\
HBME-1+, Galectin-3- & $5(31.3 \%)$ & $11(68.7 \%)$ \\
HBME-1-, Galectin-3+ & $1(100 \%)$ & 0 \\
HBME-1-, Galectin-3- & $5(50 \%)$ & $5(50 \%)$ \\
Overall & $16(27.1 \%)$ & $43(72.9 \%)$ \\
\hline
\end{tabular}

${ }^{a}$ Including follicular adenomas; $P<0.001$.

Galectin-3 on the histological specimens and all were confirmed. The selection criteria included 12 cases exhibiting a discordant expression of the antibodies of the panel, five FN/AUS and two cytologically BL.

\section{Discussion}

Thyroid nodules are a common finding in everyday clinical practice, whereas only a small percentage of them is malignant. The efficacy of FNAB in making a correct diagnosis of both malignant and BL is definitely stated in literature $(1,9,30)$. On the other hand, in those cases defined as FN, the cytologic examination is not always able to distinguish malignant or suspicious nodules requiring surgical treatment from those which can be followed-up safely.

The category of FN (Thy 3 in the BTA classification) represents an indeterminate category responsible for a high rate of unnecessary thyroidectomies (5-8). The problem may be due to the discrepancies between the cytologic and the corresponding histologic pictures because this category embraces BL showing scattered nuclear pleomorphism (e.g. hyperplastic nodules in Hashimoto's thyroiditis and toxic adenomas) and malignant lesions with moderate pleomorphism of the follicular cells (e.g. well-differentiated FC).

The cytologic criteria for diagnosing $\mathrm{FN}$ are not universally accepted and the category itself is debated. Though the cytologic picture of a follicular-patterned proliferation is well established, the diagnostic features which are helpful to assess the malignant nature of the nodule (e.g. nuclear atypia, nuclear clearing) are much less reproducible (31-33).

To address the problem of the management of these indeterminate diagnoses, immunohistochemical procedures using antibodies against antigens associated with malignancy were introduced. Among them Galectin-3 and HBME-1 have shown the highest reliability, particularly in differentiated carcinoma (10-13, 34).

The quality of the immunocytochemical reaction carried out on LBC slides is better than conventional smears in terms of morphologic details and purity of background, as some studies have demonstrated, including some from our group (17-19, 34).

The combination of the antibody panel made up of HBME-1 and Galectin-3 results in a significant increase of the diagnostic accuracy of the technique $(78.3 \%$ compared with $54.2 \%$ of the morphology alone) but this improvement turns out even more striking in FN as both the overall and the concordant panel results are considered (82 and $91.1 \%$ respectively), as is also depicted in Table 5. It is important to emphasize the relevance of an appropriate cellularity to assess the positivity or negativity of the immunocytochemical reaction. In case of scant cellularity, which is defined as not 6 groups of follicular cells, the immunocytochemical diagnosis should not be made.

An immunocytochemical-based classification of indeterminate thyroid lesions has never been attempted because of the difficulty in performing immunocytochemistry on conventional smears. Nevertheless, the uncertainty regarding the most appropriate treatment of FNs (Thy 3 in the BTA classification, ACUS and FNs in the Bethesda classification) that often sent to surgery but result in being found as benign on the definitive histology, has prompted the development of different techniques to define the cancer risk preoperatively (e.g. immunocytochemical and molecular studies (35-40)). The results of these investigations are quite promising but there is still a relevant amount of lesions that undergo unnecessary surgery or, on the other hand, may harbor an undiagnosed cancer. The current investigation achieves results similar to the molecular studies with a significantly cost-effective method still based on the morphologic evaluation of the follicular cells. The results of Nikiforov et al. $(39,40)$ compared with the current data show a better positive predictivity for the molecular analysis compared with

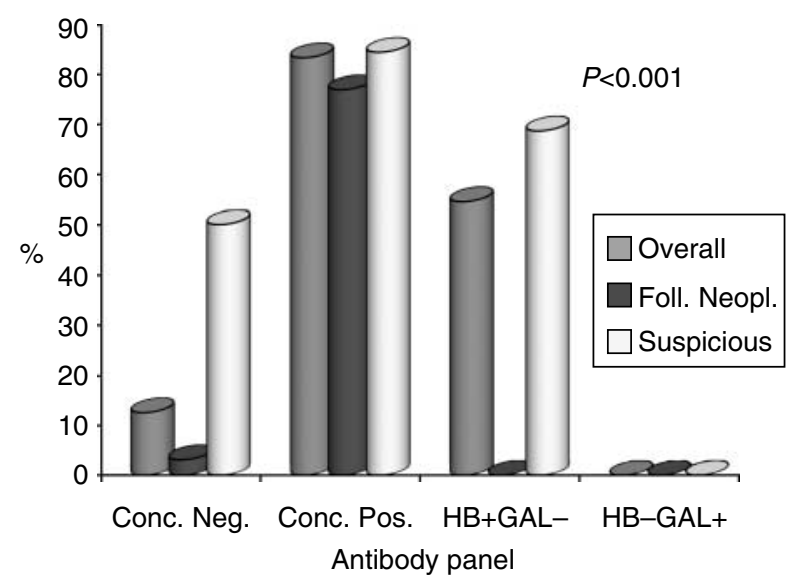

Figure 1 Malignancy rates for different antibodies combinations in the cytological categories. Conc. Neg., concordant negative panel; Conc. Pos., concordant positive panel; $\mathrm{HB}+\mathrm{GAL}-$, HBME-1 positive/Galectin-3 negative; HB-GAL +, HBME-1 negative/ Galectin-3 positive; Foll. Neopl., follicular neoplasm. 
Table 5 Descriptive statistics for the morphologic and immunocytochemical yieldings in all cytologic categories and only in follicular neoplasm lesions. Data are presented as \%.

\begin{tabular}{|c|c|c|c|c|c|c|}
\hline & \multirow[b]{2}{*}{ Cytology } & \multirow[b]{2}{*}{ ICC only ${ }^{b}$} & \multicolumn{2}{|c|}{ FN panels } & \multirow[b]{2}{*}{ HBME-1 } & \multirow[b]{2}{*}{ Galectin-3 } \\
\hline & & & All & Conc. & & \\
\hline Sensitivity & 100 & 89.7 & 91.7 & 90.9 & 72.3 & 80 \\
\hline Specificity & 12.7 & 67.7 & 78.9 & 91.2 & 88 & 72.3 \\
\hline Diagnostic accuracy & 54.2 & 78.3 & 82 & 91.1 & 80 & 76.6 \\
\hline PPV & 51 & 72.2 & 57.9 & 76.9 & 89.6 & 69 \\
\hline NPV & 100 & 87.5 & 96.8 & 96.9 & 71 & 84 \\
\hline
\end{tabular}

${ }^{a}$ Follicular neoplasms; Thy 3, and suspicious of malignancy; Thy 4, are counted as malignant; ICC, immunocytochemistry.

${ }^{\mathrm{b}}$ All combinations of antibodies where at least one of them was positive were accounted as malignant; All panels, all possible combinations of HBME-1 and Galectin-3 expressions (see Table 2); Conc. panels, HBME-1 and Galectin-3 are both positive or negative; FN, follicular neoplasm (Thy 3); PPV, positive predictive value; NPV, negative predictive value.

immunocytochemistry in the indeterminate (FN/AUS) lesions (100 vs $76.9 \%$ ) but a lower negative predictive value (16.2 vs 3.2\%). As a rule in our institution, lesions classified as FN (Thy 3) undergo immunocytochemical analysis for HBME-1 and Galectin-3 expression: those showing a simultaneous positivity for both antibodies are referred to surgery whereas those exhibiting a simultaneous negativity should be carefully followed-up, with the surgical option being held for potential future use.

Regarding the SM lesions, our results (Table 4) may look somewhat surprising but they can be easily explained. This category is usually cytologically dyshomogeneous being composed of a mixture of large atypical cells and smaller thyrocytes as is often observed in cases of FVPC. Thus, the chosen cutoff of $50 \%$ expression of HBME- 1 and Galectin- 3 may result in some false-negative cases that nonetheless do not represent a true diagnostic problem as the inclusion of a case in the SM category is mainly based on the cytologic features.

As a consequence of these results, HBME-1 has a far better diagnostic accuracy than Galectin-3 and, in case of availability of only one antibody, the use of the former as diagnostic aid in the classification of a thyroid nodule is strongly suggested.

Based on the results (Fig. 1) of the present investigation, the suggested management of a thyroid lesion undergoing FNAB includes: i) accurate examination of clinical history and serum analysis to rule out the possibility of a toxic adenoma or a Hashimoto's thyroiditis; ii) evaluation of the nuclear atypia of the follicular cells to define the inclusion of the case in the benign, FN/AUS, SM, and malignant categories; and iii) if the lesion is classified as FN/AUS (Thy 3) the assessment of the expression of HBME-1 and Galectin3 in the follicular cells would identify two additional subtypes: low risk (Thy3l or FNL - resulting HBME-1 and Galectin-3 negative) and high risk (Thy 3 h or FNH - HBME-1 and Galectin-3 positive). These subgroups are characterized by a significant difference in the risk that a $\mathrm{MN}$ is being detected at histology $(76.9 \%$ when the concordant panel is positive and $3.2 \%$ when the same panel is negative).

Therefore, the application of this antibody panel to any cytological classification that includes the category of FN/AUS may represent the first step in achieving a correct preoperative diagnosis of thyroid nodules. It improves the accuracy of the morphologic evaluation of these indeterminate lesions enabling a better selection of patients as candidates for surgery from those who should be treated pharmacologically. In this perspective, $\mathrm{LBC}$ is an improvement of conventional cytology as it represents an invaluable storage for the cellular material that can be successfully used for additional investigations.

\section{Declaration of interest}

The authors declare that there is no conflict of interest that could be perceived as prejudicing the impartiality of the research reported.

\section{Funding}

This research did not receive any specific grant from any funding agency in the public, commercial or not-for-profit sector.

\section{Acknowledgements}

The authors thank Elena Visca, Flavia Barbetti, Laura Cammarota, Alessia Maffione, and Antonella Evangelista for their invaluable technical assistance.

\section{References}

1 Gharib H \& Goellner JR. Fine-needle aspiration biopsy of the thyroid. Annals of Internal Medicine 1993118 282-289.

2 Galera-Davidson H \& Gonzalez-Campora R. Thyroid. In Comprehensive Cytopathology, ch 23, 3rd edn, pp 633-670. Eds M Bibbo \& D Wilbur. Philadelphia: Saunders Elsevier, 2008.

3 Danese D, Sciacchitano S, Farsetti A, Andreoli M \& Pontecorvi A. Diagnostic accuracy of conventional versus sonography-guided fine-needle aspiration biopsy of thyroid nodules. Thyroid $1998 \mathbf{8}$ 15-21. (doi:10.1089/thy.1998.8.15)

4 Bishop-Pitman M, Abele J, Ali SZ, Duick D, Elsheikh TM, Brooke Jeffrey R, Powers CN, Randolph G, Renshaw A \& Scoutt L. Techniques for thyroid FNA: a synopsis of the National Cancer 
Institute thyroid fine-needle aspiration state of the science conference. Diagnostic Cytopathology 200836 407-424. (doi:10. 1002/dc.20829)

5 Baloch ZW \& LiVolsi VA. Follicular-patterned lesions of the thyroid: the bane of the pathologist. American Journal of Clinical Pathology $2002 \mathbf{1 1 7}$ 143-150. (doi:10.1309/8VL9-ECXYNVMX-2RQF)

6 Baloch ZW, Fleisher S, LiVolsi VA \& Gupta PK. Diagnosis of "follicular neoplasm": a gray zone in thyroid fine-needle aspiration cytology. Diagnostic Cytopathology 200226 41-44. (doi:10.1002/ dc.10043)

7 Poller DN, Ibrahim AK, Cummings MH, Mikel JJ, Boote D \& Perry M. Fine-needle aspiration of the thyroid. Importance of an indeterminate diagnostic category. Cancer Cytopathology 2000 90 239-244. (doi:10.1002/1097-0142(20000825)90:4<239:: AID-CNCR7 > 3.0.CO;2-S)

8 Greaves TS, Olvera M, Florentine BD, Raza AS, Cobb CJ, TsaoWei DD, Groshen S, Singer P, Lopresti J \& Martin SE. Follicular lesions of thyroid. A 5-year fine-needle aspiration experience. Cancer Cytopathology 200090 335-341.

9 Baloch ZW, LiVolsi VA, Asa SL, Rosai J, Merino MJ, Randolph G, Vielh P, DeMay RM, Sidawy MK \& Frable WJ. Diagnostic terminology and morphologic criteria for cytologic diagnosis of thyroid lesions: a synopsis of the National Cancer Institute Fine-needle aspiration state-of-science conference. Diagnostic Cytopathology 200836 425-437. (doi:10.1002/dc.20830)

10 Griffith OL, Chiu CG, Gown AM, Jones SJM \& Wiseman SM. Biomarker panel diagnosis of thyroid cancer: a critical review. Expert Review of Anticancer Therapy $2008 \mathbf{8}$ 1399-1413. (doi:10. 1586/14737140.8.9.1399)

11 Bartolazzi A, Gasbarri A, Papotti M, Bussolati G, Lucante T, Khan A, Inohara H, Marandino F, Orlandi F, Nardi F, Vecchione A, Tecce R \& Larsson O. Application of an immunodiagnostic method for improving preoperative diagnosis of nodular thyroid lesions. Lancet 2001357 1644-1650. (doi:10.1016/S01406736(00)04817-0)

12 Papotti M, Volante M, Saggiorato E, Deandreis D, Veltri A \& Orlandi F. Role of galectin-3 immunodetection in the cytological diagnosis of thyroid cystic papillary carcinoma. European Journal of Endocrinology 2002147 515-521. (doi:10.1530/eje. $0.1470515)$

13 Herrmann ME, LiVolsi VA, Pasha TL, Roberts SA, Wojcik EM \& Baloch ZW. Immunohistochemical expression of galectin-3 in benign and malignant thyroid 5-year lesions. Archives of Pathology E Laboratory Medicine 2002126 710-713.

14 Miettinen M \& Kovatich AJ. HBME-1 monoclonal antibody useful in the differential diagnosis of mesothelioma, adenocarcinoma and soft tissue and bone tumors. Applied Immunohistochemistry and Molecular Morphology 19953 115-122.

15 Cheung CC, Ezzat S, Freeman JL, Rosen IB \& Asa SL. Immunohistochemical diagnosis of papillary thyroid carcinoma. Modern Pathology $2001 \quad 14$ 338-342. (doi:10.1038/modpathol. 3880312)

16 Rossi ED, Raffaelli M, Mule' A, Miraglia A, Lombardi CP, Vecchio FM \& Fadda G. Simultaneous immunohistochemical expression of HBME-1 and galectin-3 differentiates papillary carcinomas from hyperfunctioning lesions of the thyroid. Histopathology 200648 795-800. (doi:10.1111/j.1365-2559. 2006.02428.x)

17 Dabbs D, Abendroth CS, Grenko RT, Wang X \& Gail ER. Immunocytochemistry on the ThinPrep processor. Diagnostic Cytopathology $1997 \quad 17$ 388-392. (doi:10.1002/(SICI)10970339(199711)17:5<388::AID-DC14 > 3.0.CO;2-J)

18 Tabbara SO, Sidaway MK, Frost AR, Brosky KR, Coles V, Hecht S, Radcliffe G \& Sherman ME. The stability of estrogen and progesterone receptor expression on breast carcinoma cells stored as preservCyt suspension and as ThinPrep slides. Cancer Cytopathology $1998 \mathbf{8 4}$ 355-360. (doi:10.1002/(SICI)10970142(19981225)84:6<355::AID-CNCR7 > 3.0.CO;2-H)
19 Fadda G, Rossi ED, Mulè A, Miraglia A, Vecchio FM \& Capelli A. Diagnostic efficacy of immunocytochemistry on fine needle aspiration biopsies processed by thin-layer cytology. Acta Cytologica 200650 129-135. (doi:10.1159/000325920)

20 Biscotti CV, Hollow JA, Toddy SM \& Easley KA. ThinPrep versus conventional smears cytological preparations in the analysis of thyroid fine-needle aspiration 5-year specimens. American Journal of Clinical Pathology 1995104 150-153.

21 Frost AR, Sidawy MK, Ferfelli M, Tabbara SO, Bronner NA, Brosky KR \& Sherman ME. Utility of thin-layer preparations in thyroid fine-needle aspiration. Cancer Cytopathology $1998 \mathbf{8 4}$ 17-25. (doi:10.1002/(SICI)1097-0142(19980225)84:1<17:: AID-CNCR $4>3.0 .(\mathrm{CO} ; 2-\mathrm{C})$

22 Michael CW \& Hunter B. Interpretation of fine-needle aspirates processed by ThinPrep technique: cytologic artifacts and diagnostic pitfalls. Diagnostic Cytopathology 200023 6-13. (doi:10. 1002/1097-0339(200007)23:1<6::AID-DC2>3.0.CO;2-F)

23 Scurry JP \& Duggan MA. Thin layer compared to direct smear in thyroid fine-needle aspiration. Cytopathology 200011 104-115. (doi:10.1046/j.1365-2303.2000.00228.x)

24 Afify AM, Liu J \& Al-Khafaji BM. Cytologic artifacts and pitfalls of thyroid fine-needle aspiration using ThinPrep. Cancer Cytopathology 200193 179-186. (doi:10.1002/cncr.9027)

25 Cochand-Priollet B, Prat JJ, Polivka M, Thienpont L, Dahan H, Wassef M \& Guillausseau PJ. Thyroid fine needle aspiration: the morphological features on ThinPrep slide preparations. Eighty cases with histological control. Cytopathology 200314 343-349. (doi:10.1046/j.0956-5507.2003.00098.x)

26 Fadda G, Rossi ED, Raffaelli M, Mulè A, Pontecorvi A, Miraglia A, Lombardi CP \& Vecchio FM. Fine-needle aspiration biopsy of thyroid lesions processed by thin-layer cytology: one-year institutional experience with histologic correlation. Thyroid 200616 975-981. (doi:10.1089/thy.2006.16.975)

27 Rossi ED, Raffaelli M, Zannoni GF, Pontecorvi A, Mulè A, Callà C, Lombardi CP \& Fadda G. Diagnostic efficacy of conventional as compared to liquid-based cytology in thyroid lesions. Evaluation of 10,360 fine needle aspiration cytology cases. Acta Cytologica 2009 53 659-666. (doi:10.1159/000325407)

28 British Thyroid Association Guidelines for the Management of Thyroid Cancer. 2nd edn, 2007.

29 Cooper DS, Doherty GM, Haugen BR, Kloos RT, Lee SL, Mandel SJ, Mazzaferri EL, McIver B, Pacini F, Schlumberger M, Sherman SI, Steward DL \& Tuttle RM. Revised American Thyroid Association management guidelines for patients with thyroid nodules and differentiated thyroid cancer. Thyroid 200919 1-48. (doi:10. 1089/thy.2009.0110)

30 Ravetto C, Colombo L \& Dottorini ME. Usefulness of fine-needle aspiration in the diagnosis of thyroid carcinoma. A retrospective study in 37,895 patients. Cancer Cytopathology $2000 \mathbf{9 0}$ 357-363. (doi:10.1002/1097-0142(20001225)90:6<357:: AID-CNCR6 > 3.0.CO;2-4)

31 Nga ME, Kumarasinghe MP, Tie B, Sterrett GF, Wood B, Walsh J, Nguyen H, Whyte A \& Frost FA. Experience with standardized thyroid fine-needle aspiration reporting categories. Cancer Cytopathology 2010118 423-433 (published online). (doi:10.1002/ cncy.20111)

32 Yang J, Schnadig V, Logrono R \& Wassermann PG. Fine-needle aspiration of thyroid nodules: a study of 4703 patients with histologic and clinic correlations. Cancer $2007111306-315$. (doi:10.1002/cncr.22955)

33 Faquin WC \& Baloch ZW. Fine-needle aspiration of follicular patterned lesions of the thyroid: diagnosis, management and follow-up according to NCI recommendations. Diagnostic Cytopathology 201038 731-739. (doi:10.1002/dc.21292)

34 Rossi ED, Raffaelli M, Minimo C, Mule' A, Lombardi CP, Vecchio FM \& Fadda G. Immunocytochemical evaluation of thyroid neoplasms on thin-layer smears from fine-needle aspiration biopsies. Cancer Cytopathology 2005105 87-95. (doi:10.1002/cncr. 21026) 
35 Sack MJ, Astengo-Osuna C, Lin BT, Battifora H \& LiVolsi VA. HBME-1 immunostaining in 5-year thyroid fine-needle aspirations: a useful marker in the diagnosis of carcinoma. Modern Pathology 199710 668-674.

36 Beesley MF \& McLaren KM. Cytokeratin 19 and galectin-3 immunohistochemistry in the differential diagnosis of solitary thyroid nodules. Histopathology 200241 236-243. (doi:10.1046/ j.1365-2559.2002.01442.x)

37 Saggiorato E, De Pompa R, Volante M, Cappia S, Arecco F, Dei Tos AP, Orlandi F \& Papotti M. Characterization of thyroid "follicular neoplasms" in fine-needle aspiration cytologic specimens using a panel of immunohistochemical markers: a proposal for clinical application. Endocrine-Related Cancer 200512 305-317. (doi:10.1677/erc.1.00944)

38 Schmitt FC, Longatto-Filho A, Valent A \& Vielh P. Molecular techniques in cytopathology practice. Journal of Clinical Pathology 200861 258-267. (doi:10.1136/jcp.2006.044347)
39 Nikiforov YE, Steward DL, Robinson-Smith TM, Haugen BR, Klopper JP, Zhu Z, Fagin JA, Falciglia M, Weber K \& Nikiforova MN. Molecular testing for mutations in improving the fine-needle aspiration diagnosis of thyroid nodules. Journal of Clinical Endocrinology and Metabolism 200994 2092-2098. (doi:10. 1210/jc.2009-0247)

40 Ohori NP, Nikiforova MN, Schoedel KE, LeBeau SO, Hodak SP, Seethala RR, Carty SE, Ogilvie JB, Yip L \& Nikiforov YE. Contribution of molecular testing to thyroid fine-needle aspiration cytology of "follicular lesion of undetermined significance/atypia of undetermined significance". Cancer Cytopathology 2010118 17-23. (doi:10.1002/cncy.20063)

Received 21 April 2011

Accepted 1 July 2011 